\title{
The coexistence of ankylosing spondylitis and diffuse idiopathic skeletal hyperostosis
}

\author{
M. Wooten
}

Received: 20 January 2009 / Accepted: 27 January 2009/Published online: 18 February 2009

(C) Clinical Rheumatology 2009

Dear Editor,

I have read with interest the article by Xavier Jordana and colleagues entitled "The Coexistence of Ankylosing Spondylitis and Diffuse Idiopathic Skeletal Hyperostosis-A Postmortem Diagnosis" (Clinical Rheumatology, 2008; DOI 10.1007/s10067-008-1068-9). The authors point out the rarity of these two disorders coexisting.

The authors mention two cases reported in the literature regarding these two disorders, those of Kozanoglu et al. and
Moreno et al. It should be noted, however, that a total of at least 15 cases have been reported in the literature and that theirs would be at least the sixteenth such case (Wooten MD, Canino VO, "Diffuse Idiopathic Skeletal Hyperostosis" Federal Practitioner, 2003; 20:66-75). A patient need not be HLA-B27-positive to make this diagnosis. While the coexistence of these two disorders still remains a relatively rare phenomenon, it should be considered in patients who manifest signs and symptoms of both disorders.

M. Wooten $(\triangle)$

Department of Veterans Affairs,

Northern Indiana Health Care System,

Fort Wayne, IN, USA

e-mail: marc.wooten@med.va.gov

M. Wooten

Indiana University School of Medicine,

Indiana, IN, USA 